\title{
Symposium Participants
}

\section{GHANA}

\section{Ms Helena Awurasa}

Co-ordinator

Ghana National Association of Teachers

National Gender and EFAIDS

PO Box 209

ACCRA

Tel: +23321221515

e-mail: awurasa@yahoo.co.uk

\section{KENYA}

\section{Ms Lucy Barimbui Njura}

Programme Co-ordinator

Kenya National Union Of Teachers

EFAIDS

PO Box 30407 — 00100

NAIROBI

Tel: +25420223849

Fax: +442542022 2701

e-mail: knut@nbnet.co.ke

\section{MOZAMBIQUE}

\section{Dr Teodora Magaia Cassamo}

Head

School Health and HIV/AIDS Department

Ministry of Education and Culture

Av 24 Julho 164

MAPUTO

Tel: + 2582121492461

Fax: + 25821214010979

e-mail: teodora.cassamo@mec.gov.mz

\section{Mr Messias Matusse}

Director

Estrada Nacional No1

Marracuene

MAPUTO

Tel: + 25821759180

Fax: + 25821759177

e-mail: messias@mec.gov.mz

\section{Mrs Esameralda Aurelio Mutemba}

Head

Gender, Community Involvement Department

Ministry of Education and Culture

Av 24 de Julho 167

MAPUTO

Tel: + 25821492461

Fax: + 25821492196

e-mail: emutemba@mec.gov.mz

\section{Ms Naima Sau}

Deputy Director for Human Resources

Ministry of Education and Culture

Av 24 de Julho 167

MAPUTO

Mob: + 258848343893

e-mail: naima@mec.gov.mz

\section{Ms Aissa Braga}

Head

Special Education

Ministry of Education

Av 24 de Julho 164

MAPUTO

Tel: + 25821491658

e-mail: aissa@mec.gov.mz

Dr Aboubacar Omar Ibrahimo

Deputy National Director TEVT

Ministry of Education and Culture

Av 24 de Julho 167

MAPUTO

Tel: +25821490192

e-mail: aomar@mec.gov.mz 
Ms Emelina Ana Khossa

Assistant in High Education

Ministry of Education

Av 24 de Julho 167

MAPUTO

Tel: +258 21491142

Fax: + 25821491042

e-mail: emelina.khossa@mec.gov.mz

\section{Mr Armando Francisco Sambo}

Co-operation Agent

Ministry of Education

Av 24 de Julho 164

MAPUTO

Tel: + 258825389648

e-mail: asambo@mec.com

\section{Ms Zaida Baule}

Desk Officer

Ministry of Education and Culture

Av 24 de Julho 167

P 0 Box 34

MAPUTO

Tel: +25821490892

Fax:

e-mail: zbaule@mec.gov.mz

Mr Manuel Zianja Guro

Ministry of Education and Culture

Rua Comandante Augusto Cardoso 81

P 0 Box 4653

MAPUTO

Tel: + 25821426797

Fax: +258 21426797

e-mail: babyfan66@yahoo.com

\section{Professor Inocente Mutimucuio}

Eduardo Mondlane University

CP 257

MAPUTO

Tel: +25821495422

Fax: + 25821493313

e-mail: inovasco@zebra.uem.mz

\section{Professor Marcos Cherinda}

Head

Research Department

Universidade Pedagogica

MAPUTO

Tel: +25821400269

Fax: +258 21400269

e-mail: cherinda@tvcabo.co.mz

\section{Mr Diogo Milagre}

Deputy Executive Secretary

Mozambique National Aids council

Rua Antonio Bocarro no 106-144

MAPUTO

Tel: +258 $21495604 / 5$

Fax:

e-mail: diogo.milagre@cncs.org.mz

\author{
National Organisation of Professors \\ Mr David Uassane Chinavane \\ Secretary \\ International Relations Department \\ Organizacao Nacional dos Professores \\ National Headquarters \\ Av Eduardo Mondlane 49 \\ MAPUTO \\ Tel: + 25821490400 \\ Fax: + 25821490400 \\ e-mail: david.chinavane@yahoo.com.br
}

\section{Ms Aida Joaquim Munhequete}

Teacher Trainer

Institute of Languages

ONP

MAPUTO

Tel: + 25821425684

Fax:

e-mail: amunhequete@gmail.com

\section{Mr Charles Janisse}

ONP Member

E P C Laulane

MAPUTO

Tel: + 258820972680

Fax: 25821490400

e-mail: onpsnpmc@tvcabo.co.mz 


\section{SOUTH AFRICA}

Ms Lulama Nare

Head of Department

Gender Unit

South African Democratic Teachers Union

49 Goud Cnr Marshal

JOHANNESBURG

Tel: + 27113344830

Fax: + 27113344834

e-mail: Inare@sadtu.org.za

\section{UNITED KINGDOM}

\section{Mr Richard Bourne}

Senior Research Fellow

Institute of Commonwealth Studies

28 Russell Square

LONDON WC1B 5DS

Tel: +44 2078628823

Fax: +44 2078628820

e-mail: Richard.Bourne@sas.ac.uk

Dr Paul Miller

Head

Business and Economics Department

Highlands School

148 World's End Lane

Enfield

LONDON N21 1QQ

Tel: +44 2083701100

Fax:+ 442083701110

e-mail: ojuko76@yahoo.com

\section{ZAMBIA}

\section{Professor Michael Kelly}

Consultant

Luwisha House

5880 Gt East Road

P 0 Box 35391

LUSAKA

Tel: + 260211291606

Fax: + 260211293763

e-mail: mjkelly@jesuits.org.zm

\section{COMMONWEALTH TEACHERS GROUP}

Ms Samidha Garg

Principal Officer

International Relations

National Union of Teachers

Hamilton House

Mabledon Place

LONDON WC1H 9BD

Tel: +44207380 4716

Fax: +44 2073884857

e-mail: s.garg@nut.org.uk

\section{EDUCATION INTERNATIONAL}

\section{Mr Dennis Sinyolo}

Co-ordinator

Education and Employment

5 Bd du Ropi Albert II

1210 BRUSSELS

Tel: +32 495339559

Fax: +32 22240606

e-mail: dennis.sinnyolo@ei-ie.org

\section{UNESCO}

Mr Benoit Sossou

Representative

P 0 Box 1397

MAPUTO

Tel: +25821481702

Fax: +258 21493431

e-mail: b.sossou@unesco.org

\section{COMMONWEALTH SECRETARIAT}

Dr Rolande Degazon-Johnson

Adviser (Education)

Marlborough House

Pall Mall

LONDON SW1Y 5HX

Tel: +44 2077476289

Fax: +44 2077476287

e-mail: r.degazon-johnson@commonwealth.int 